ANT/EP/CP-76192

DE93 002928

\title{
Laser Beam Diagnostics for Kilowatt Power Pulsed YAG Laser
}

\author{
Yi Liu and Keng H. Leong \\ Laser Applications Laboratory \\ Engineering Physics Division \\ Argonne National Laboratory \\ Argonne, IL 60439, USA
}

Extended abstract for ICALEO'92 Proceedings
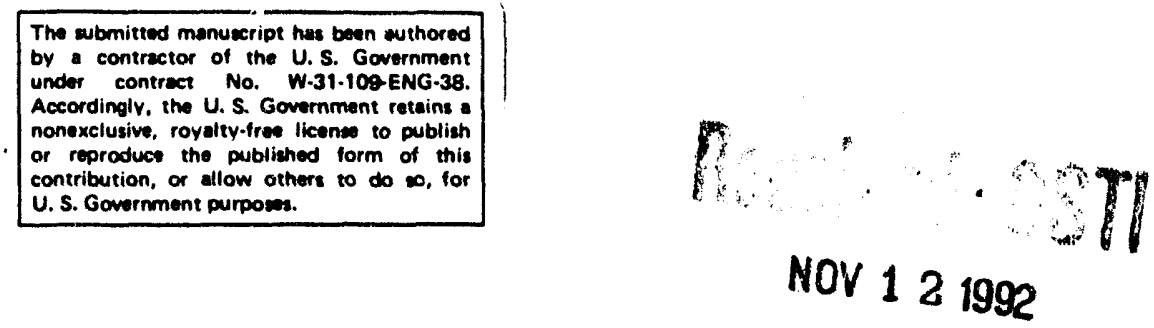

\section{DISCLAIMER}

This report was p pared as an account of work sponsored by an agency of the United States Government. Neither the United States Government nor any agency thereof, nor any of their employees, makes any warranty, express or implied, or assumes any legal liability or responsibility for the accuracy, completeness, or usefulness of any information, apparatus, product, or process disclosed, or represents that its use would not infringe privately owned rights. Reference herein to any specific commercial product, process, or service by trade name, trademark, manufacturer, or otherwise does not necessarily constitute or imply its endorsement, recommendation, or favoring by the United States Government or any agency thereof. The views and opinions of authors expressed berein do not necessarily state or reflect thoee of the United States Government or any agency thereof.

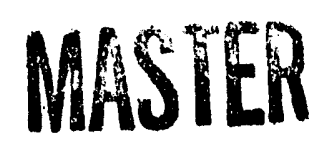




\title{
Laser Beam Diagnostics for Kilowatî Power Pulsed YAG Laser
}

\author{
Yi Liu and Keng $\mathrm{H}$. Leong \\ Laser Applications Laboratory \\ Engineering Physics Division \\ Argonne National Laboratory \\ Argonne, IL 60439, USA
}

\begin{abstract}
There is a growing need for high power YAG laser beam diagnostics with the recent introduction of such lasers in laser material processing. In this paper, we will describe the use of a commercially available laser beam analyzer (Prometec) to profile the laser beam from a $1600 \mathrm{~W}$ pulsed Nd:YAG laser that has a $1 \mathrm{~mm}$ fiber optic beam delivery system. The selection of laser pulse frequency and pulse width for the measurement is discussed. Laser beam propagation parameters by various optical components such as fibers and lenses can be determined from measurements using this device. The importance of such measurements will be discussed.
\end{abstract}

\section{Introduction}

For many years, material processing by industrial Nd:YAG lasers has been limited by the available power output. As a result, the major applications of YAG lasers in material processing are focused on drilling, spot welding, marking and electronics fabrication ${ }^{1}$ where high average power levels are not required. However, with the recent introduction of the kilowatt-level YAG lasers, high speed YAG laser cutting, welding and surface heat treat comparable to that achieved by the $\mathrm{CO}_{2}$ laser is now feasible 2 . Most metals have better absorption at the shorter wavelength YAG laser beams than $\mathrm{CO}_{2}$ laser beams at temperatures below their melting points. Hence, kilowatt YAG lasers offer processing rates and capabilities that are competitive with the multi-kilowatt $\mathrm{CO}_{2}$ lasers particularly for those processes that do not require melting or evaporation. An added benefit of the YAG laser is the ease in process automation with flexible fiber optic beam delivery. Other advantages using the YAG laser are inexpensive optics and solid state operation.

The quality of the laser processed part is impacted on by the characteristics of the laser beam. The characteristics of the YAG laser beam are determined by the laser cavity desion, the working condition of the rods, the method of launching the beam into the fiber and the focusing optics. Consequently the quality or characteristics of the laser beam delivered to the workpiece need to be determined for optimization of a beam delivery system and for quality assurance of material processing.

Many concepts and techniques have been developed in the past decade for high power laser beam profiling. The simplest methods include burn marks on plexiglass for $\mathrm{CO}_{2}$ and on Zap-it paper for YAG. The more complicated techniques are those employing scanning techniques such as Spoke wheel or needle wheel instruments 3,4 . The commercially available laser beam analyzer by Prometec (marketed in U.S. through Trumpf Industrial Lasers, Inc. and Utilase Systems, Inc.) utilizes a pinhole in a rotating hollow needle to scan through the laser beam. In this paper, we will demonstrate the ability and limitations of using the Prometec laser beam analyzer (LBA) to profile a high power pulsed YAG laser system. 


\section{Equipment Description}

A $1600 \mathrm{~W}$ pulsed Nd:YAG laser was recently installed at Argonne National Laboratory for research and development in laser aided material processing. The laser has four Nd:YAG rods in an oscillator amplifier configuration. The original laser system produced two $800 \mathrm{~W}$ laser beams that were launched into a $1 \mathrm{~mm}$ fiber. The two beams can be fired simultaneously or alternately. A new version of the laser utilizes a beam splitter to combine the beams from the two oscillators before amplification. The combined beam from the two oscillators is amplified by two in-line amplifiers and then launched into the fiber. One of the advantages of the new version is the elimination of optical apertures used in the two beam system. Most of our beam diagnostics measurements are performed on the redesigned laser. Some data on the cld design will also be discussed. The laser pulse width can be controlled between 0.5 millisecond to 20 millisecond. The laser has a pulse frequency range of 1 to $800 \mathrm{~Hz}$. The fiber used for laser beam delivery is a 1 $\mathrm{mm}$ step indexed fused silica fiber. The YAG laser beam is launched into the fiber by a short focal length lens. The output of the fiber is collimated and then shaped using appropriate optics for a particular application.

The schematic of the beam profiling experimental set up is illustrated in Figure 1. The Prometec laser beam analyzer senses the laser beam with the aid of a rotating hollow needle (Fig. 2). A pinhole is drilled near the end of the needle, in the side facing the beam. Photons admitted via the pinhole are directed to a pyroelectric detector by two inclined mirrors. As the needle carrier revolves, the beam is scanned along the path $(x)$. The axis of rotation $(y)$ is displaced by slide action, permitting the entire cross-sectional area of the beam to be sensed. The intensity distribution of a laser beam is measured by recording a cross-sectional area of the beam as it is traversed by the needle. On completion of a traverse, the feed slide moves to the next position. During the next traverse, a further cross-sectional area is sensed.

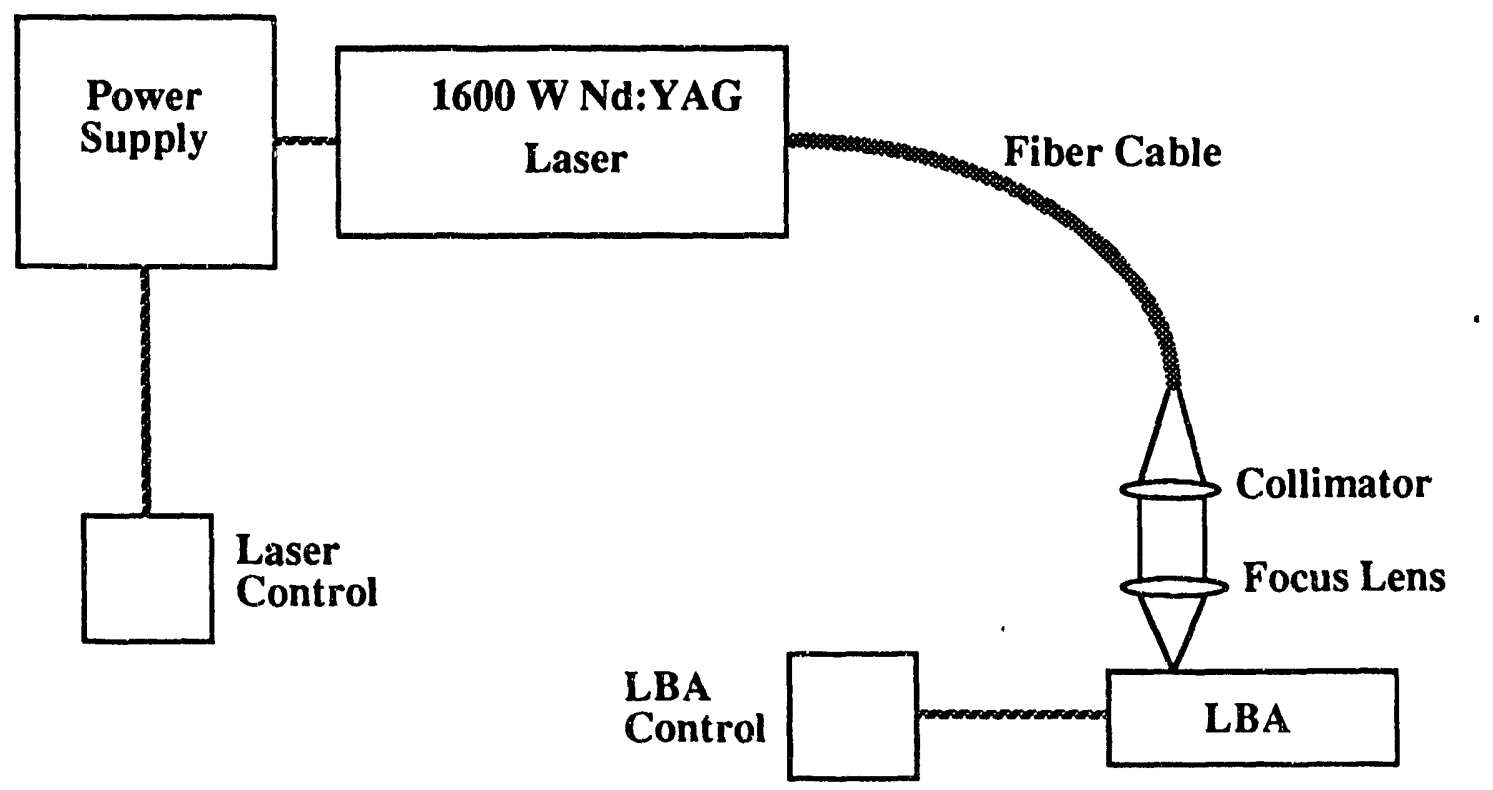

Figure 1. Schematic of laser beam diagnostics set up

Different pinhole sizes are used for different beam intensities. Usually, unfocused beams are profiled by pinholes which have diameters larger than 100 microns. The 20 or 50 microns pinholes are used to measure the focused beam. The resolution of the measurement depends on the sampling rate and the size of scanned window. The resolution varies from $75 \mu \mathrm{m}$ to $1.5 \mathrm{~mm}$ for 
different size unfocused beams and $6 \mu \mathrm{m}$ to $100 \mu \mathrm{m}$ for different focused beams. An appropriate pyroelectric detector was used for the detection of the YAG laser wavelength.

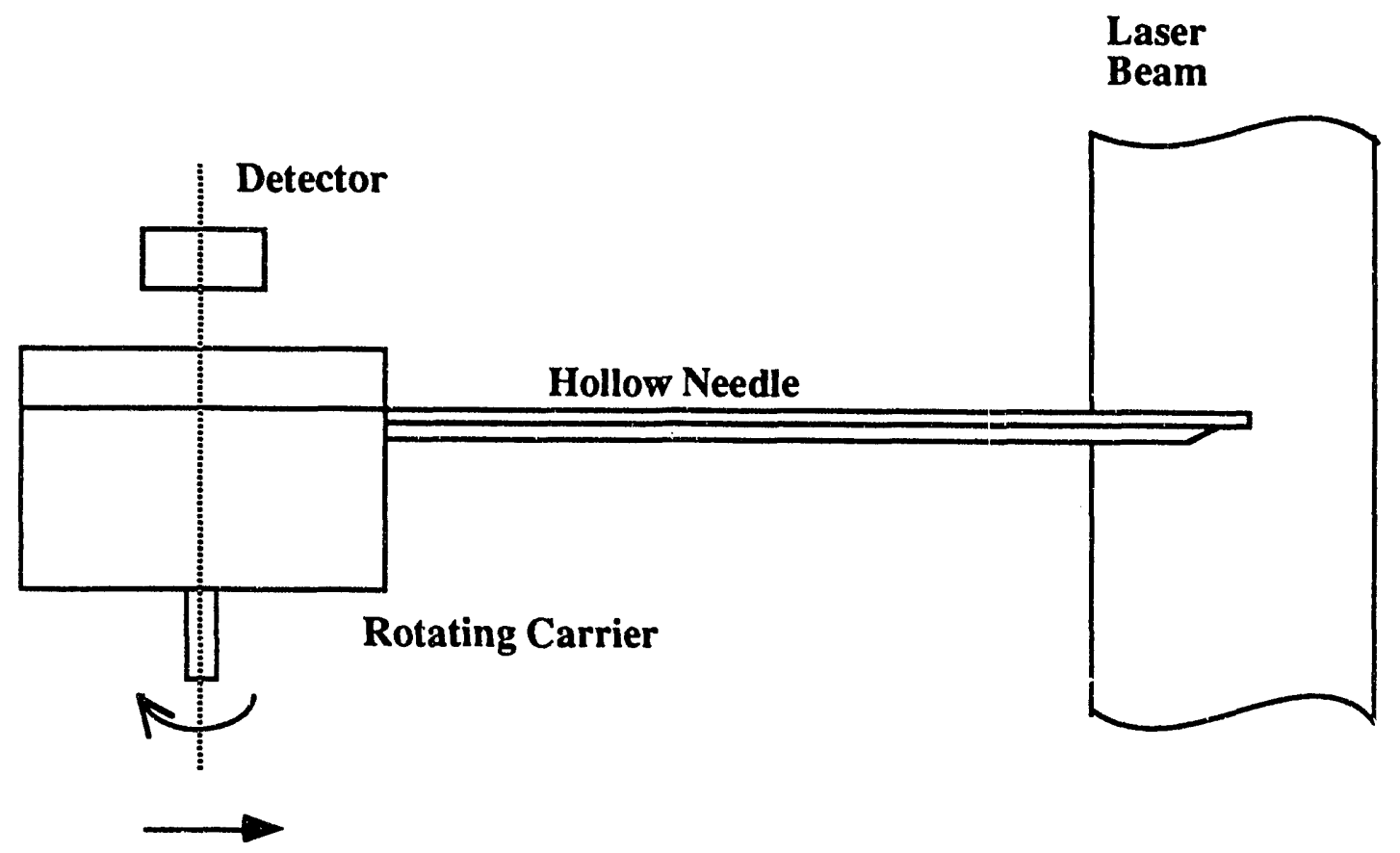

Slide Feed (y-axis)

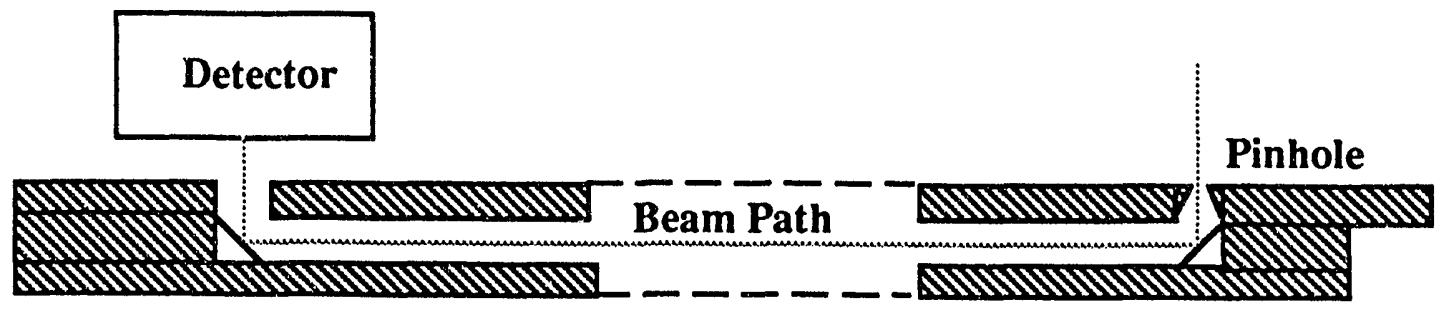

Hollow Needle

Fig. 2 Working principle of Prometec laser beam analyzer

\section{Pulsed Beam Measurements}

The LBA was intended for measuring $\mathrm{cw}$ laser beams. Careful selections of pulse parameters to match the frequency of the rotating hollow needle of the LBA allow pulse beams to be profiled. The line frequency of the LBA, i.e. the $y$-axis feed is $25 \mathrm{~Hz}$. To avoid the undesirable interference between the sampling frequency of the LBA and the pulse frequency of the laser, the laser pulse frequency needs to be an integral multiple of $25 \mathrm{~Hz}$. On the other hand, the laser pulse width should be longer than the scanning time of the hollow needle of the LBA. The tangential speed of the hollow needle for the unfocused beam is about $16 \mathrm{~m} / \mathrm{s}$. It will take about 1 millisecond to scan through a $16 \mathrm{~mm}$ diameter beam. Therefore, a pulse width of longer than 1 
millisecond is usually required for unfocused beams. For the same reason, a 0.5 millisecond or longer pulse width is needed for focused beam measurements. To complete a 2-dimensional profile, the hollow needle scans 41 arcs along y-direction and 41 separate pulses are detected. A profile along the $y$-direction will depend on the stability of the laser. Since the LBA does not have the option of synchronizing the scanning to the laser pulsing, a higher duty cycle pulse firing is required for the measurement and subsequently one successful measurement is obtained after several attempts.

\section{Results and Discussions}

A typical beam intensity distribution of the raw beam exiting the fiber is shown in Figure 3 . Figure 3(a) shows the area of beam which has $86 \%$ of the total beam energy. For a Gaussian distribution beam, the intensity at the edge of this area is about $13.5 \%$, i.e. $1 / \mathrm{e}^{2}$ of peak intensity. Two sections through the intensity distribution parallel to the $\mathrm{x}$ and $\mathrm{y}$ axes respectively are also displayed. A 3-dimensional display of the intensity distribution is shown in Figure 3(b). The energy distribution resembles a Gaussian type. However, the area in Figure 3(a) seems have two symmetrical axis along directions rotated $45^{\circ}$ from the $\mathrm{x}$ and $\mathrm{y}$ axis. This symmetry is consistent in many of our measurements and is not believed to be a sampling effect.

Although there are many methods which can be used to define the beam width ${ }^{5}$, the proposed ISO/TC standards ${ }^{6}$ prefer the enriched energy definition. This method uses a variable aperture to determine the area of the laser beam which has $86 \%$ of the total beam energy. The radius of a circle having the same area is defined as the radius of the beam. We use the radius of a circle that has the same area in Fig. 3(a) to represent the beam radius. The divergence of the beam exiting the fiber can be determined by profiling the beam at various distances from the fiber end. This result is displayed in Figure 4. The beam diameters determined by measuring the size of burn marks at various distances on Zap-it paper are also shown. Burn marks are usually bigger than the real beam sizes. Our results show a $5 \mathrm{~mm}$ increase in beam size for Zap-it paper measurements. The beam divergences calculated using both data sets are consistent and the full angle of the divergence is about 0.16 radian. The numerical aperture of the fiber used is 0.2 which corresponds to a full angle divergence of 0.4 radian. The angle of the beam convergence when it is launched into the fiber is about 0.32 radian. Our results suggest that the divergence of the beam exiting the fiber is not controlled by the numerical aperture of the fiber. Further stidy is needed to determine the factors that affect the exiting beam divergence.

Different laser beam characterics are required for different material processing applications. These requirements can be met by using different focusing optics. The focusing optic assembly for a fiber delivered YAG laser beam consists of two components. The first component is an collimator (an upcollimator may be added if necessary). In one of the optics tested, two planoconvex lenses were used as the collimator. The beam distribution after the collimators is shown in Figure 5. The area of the collimated beam which has $86 \%$ of the total beam energy still retains the symmetrical characteristics shown in the raw beam although axis of symmetry have changed. The energy profiles along the two orthogonal cross sections show that the optics has broaden the intensity distribution of the collimated beam.

The collimated beam can then be focused for different applications. In our cutting optics, two plano-convex lenses were used to focus the beam. Using a $20 \mu \mathrm{m}$ pinhole in the hollow needle, the focused beam profile was measured and the data are displayed in Figure 6 . The focused beam resembles a top hat type distribution. By profiling the focused beams at various distances from the focusing optics, the beam propagation properties can be accurately obtained. The focused beam diameters at the vicinity of the focal plane are displayed in Figure 7 . From this figure, a minimum focused beam diameter of about 600 microns was determined. The distance of the focal plane from the last surface of the optics can also be accurately determined. This distance 


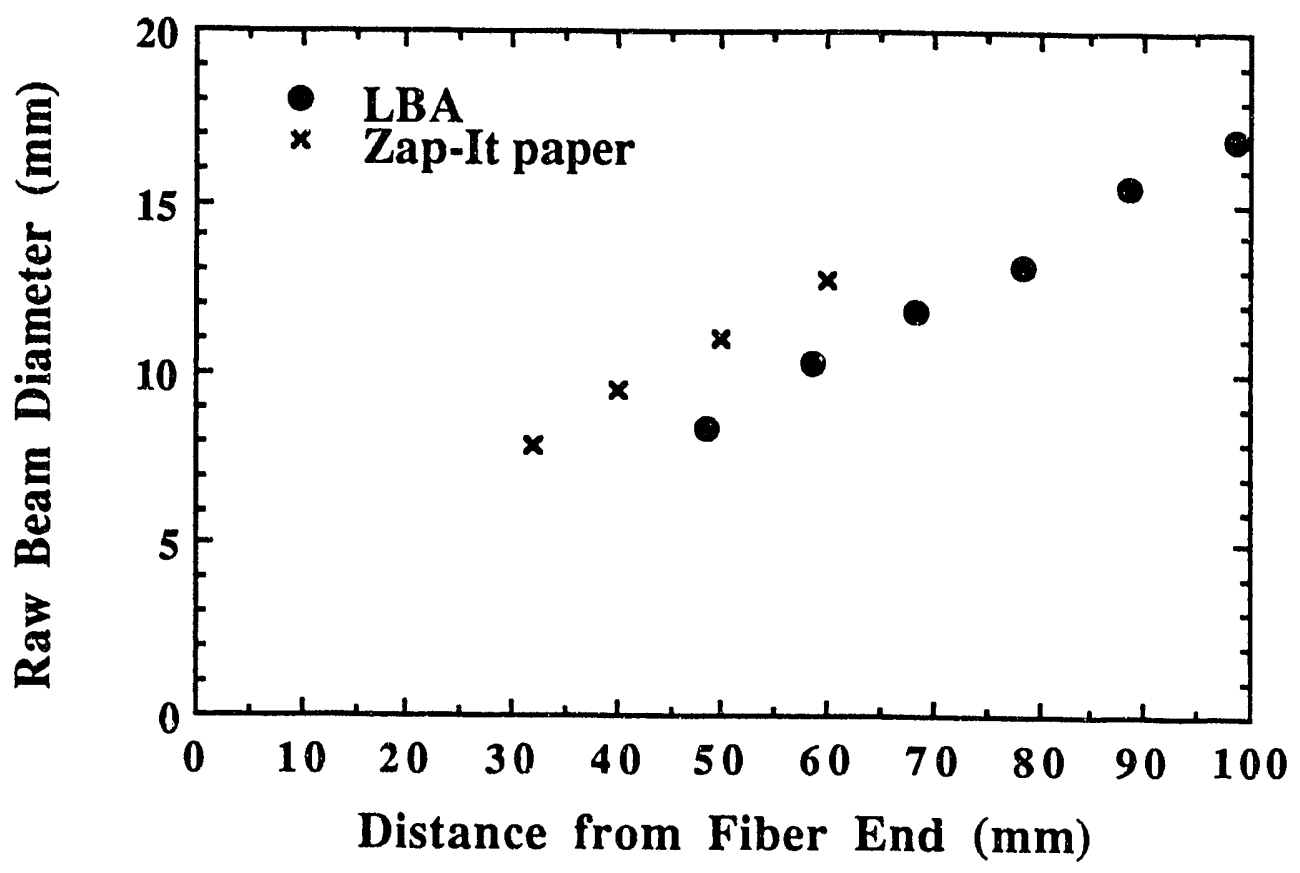

Fig. 4 Raw beam diameters measured at various distances by LBA and burn marks on Zap-it paper

is $36.5 \mathrm{~mm}$ for the tested cutting optics.

The burn hole size on a kapton polymer tape (Permacel tape) is commonly used in practice to measure the focused beam dianeter. Figure 7 also shows the focused beam size determined by this method. At higher beam intensities (smaller beam size), the burn hole sizes on Permacel tape were $25 \%$ grater than the LBA measurements. The differences become negligible when the beam intensity decreases. The depth of focus, which is a very important parameter for many laser material processing applications, is defined as the distance over which the focal spot size changes by $\pm 5 \%$. The cutting optics tested in Fig. 7 show such a value of approximately $0.4 \mathrm{~mm}$.

The variation of beam diameter with pulse energy and pulse width were examined using the LBA. The beams were profiled after the collimator and the results are shown in Figure 8. The beam sizes increase slightly with energy and pulse width. However, the changes in both cases are found to be less than $10 \%$. The possible reason for the increase may be the thermal lensing effect of the YAG rods caused by the higher pulse energy or the longer pulse width.

The early version of the Electrox $1.6 \mathrm{~kW}$ laser has two separate beams. The two beams are launched into the fiber with similar angles to the center axis of the fiber. We found that this configuration gives rise to a different beam profile from each beam. Figure 9 shows the top hat distribution obtained from one beam and a TEM $01 *$ type distribution that is from the other beam. It is generally known that the energy distribution depends on launch angle. Further study on this phenomenon is needed. 


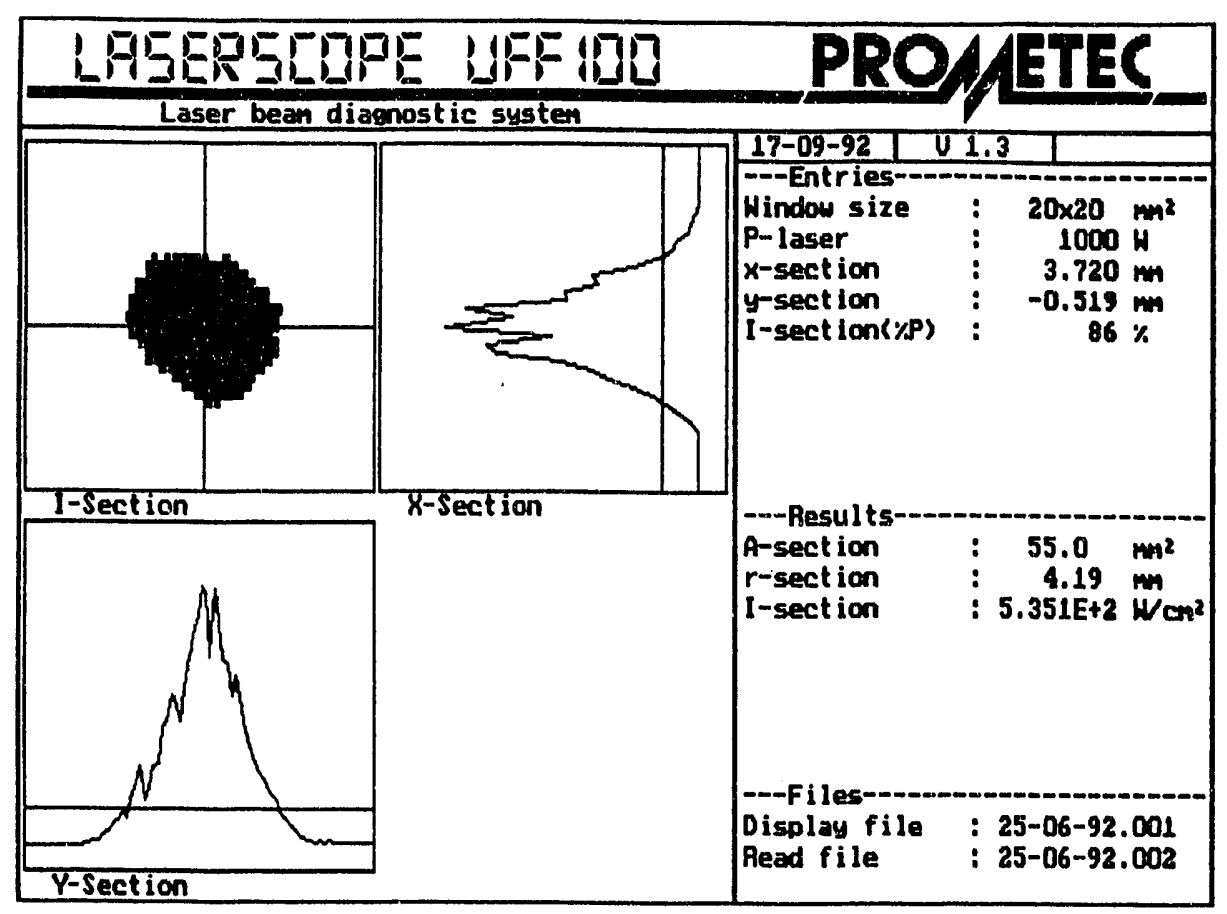

(a)

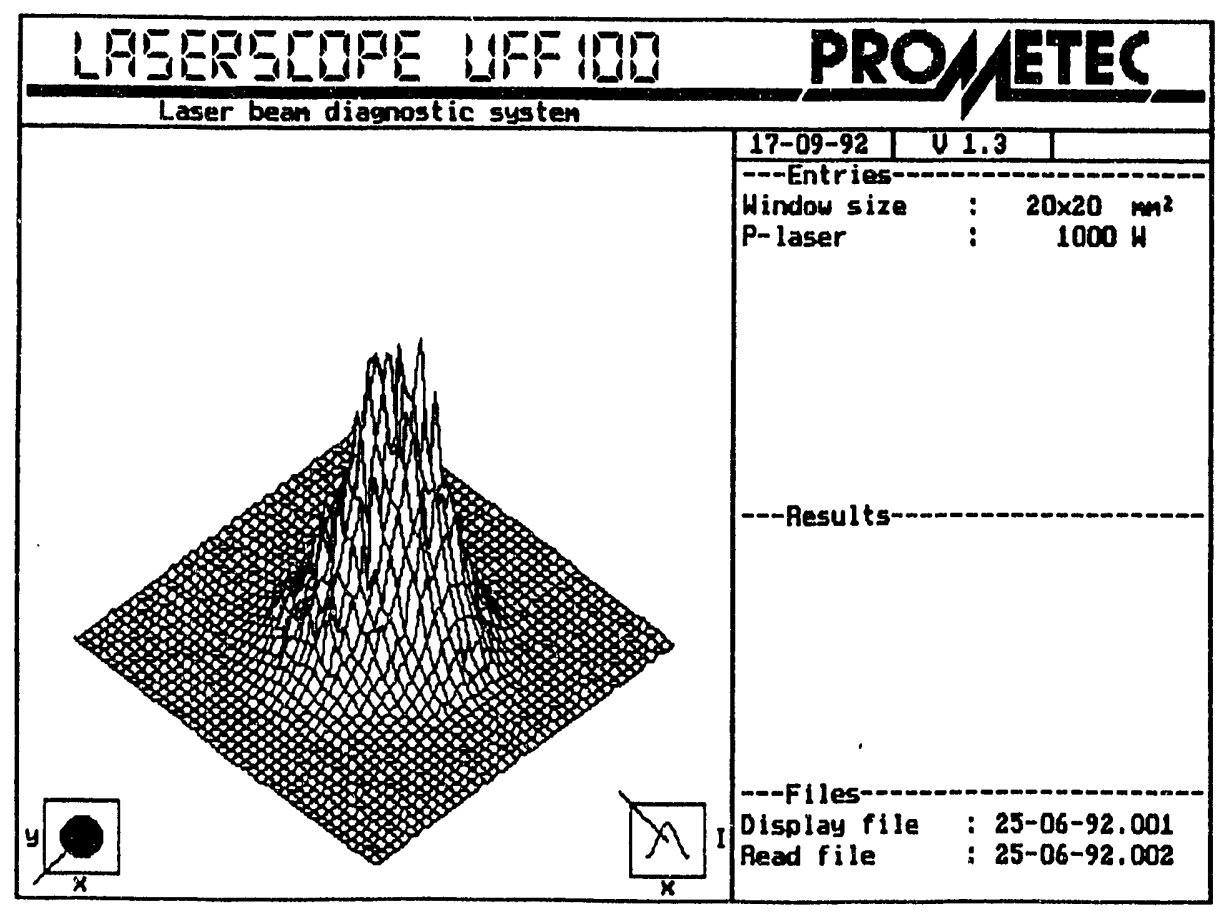

(b)

Fig. 3 Profile of the beam exiting the fiber. (a) Beam area which contains $86 \%$ of beam energy and two orthogonal cross sections; (b) Isometric representation of beam intensity distribution. 


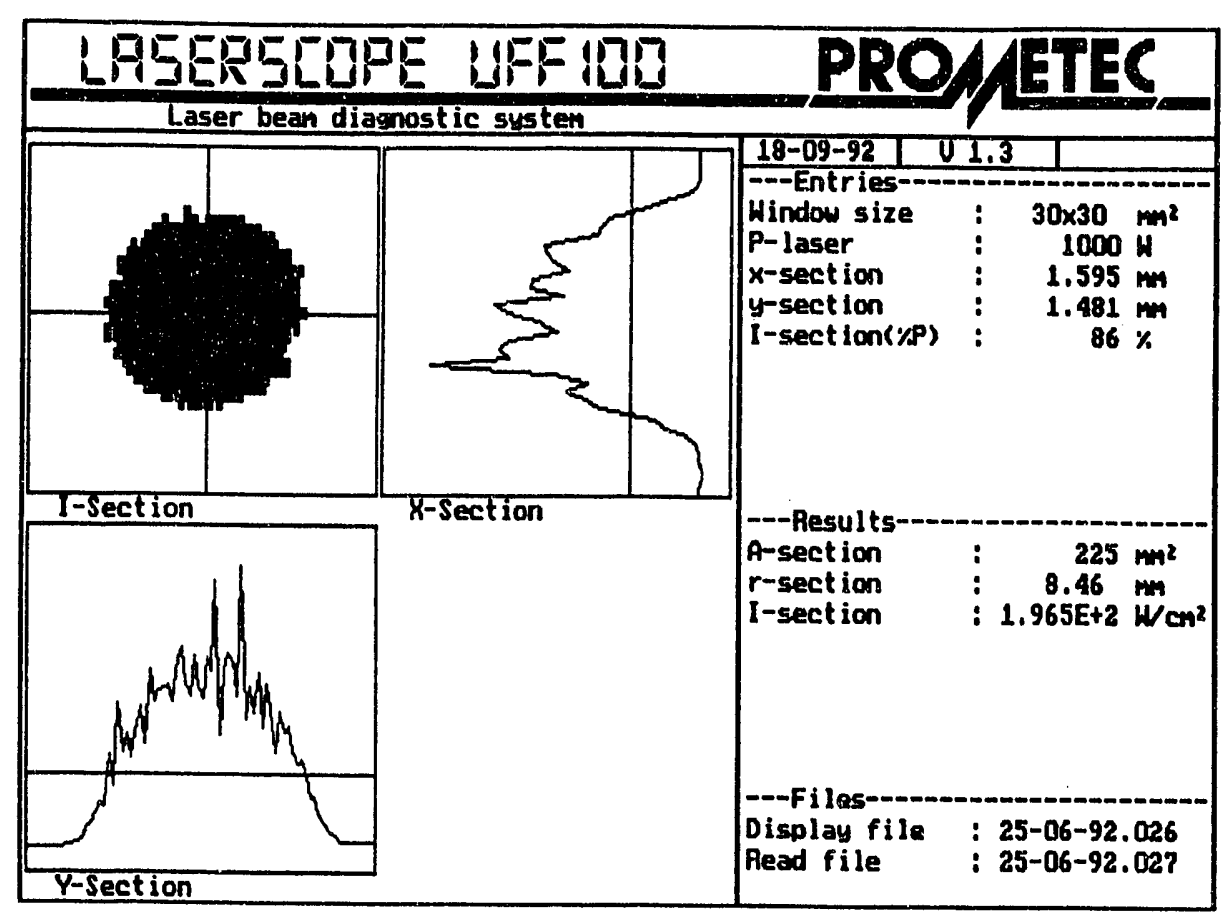

(a)

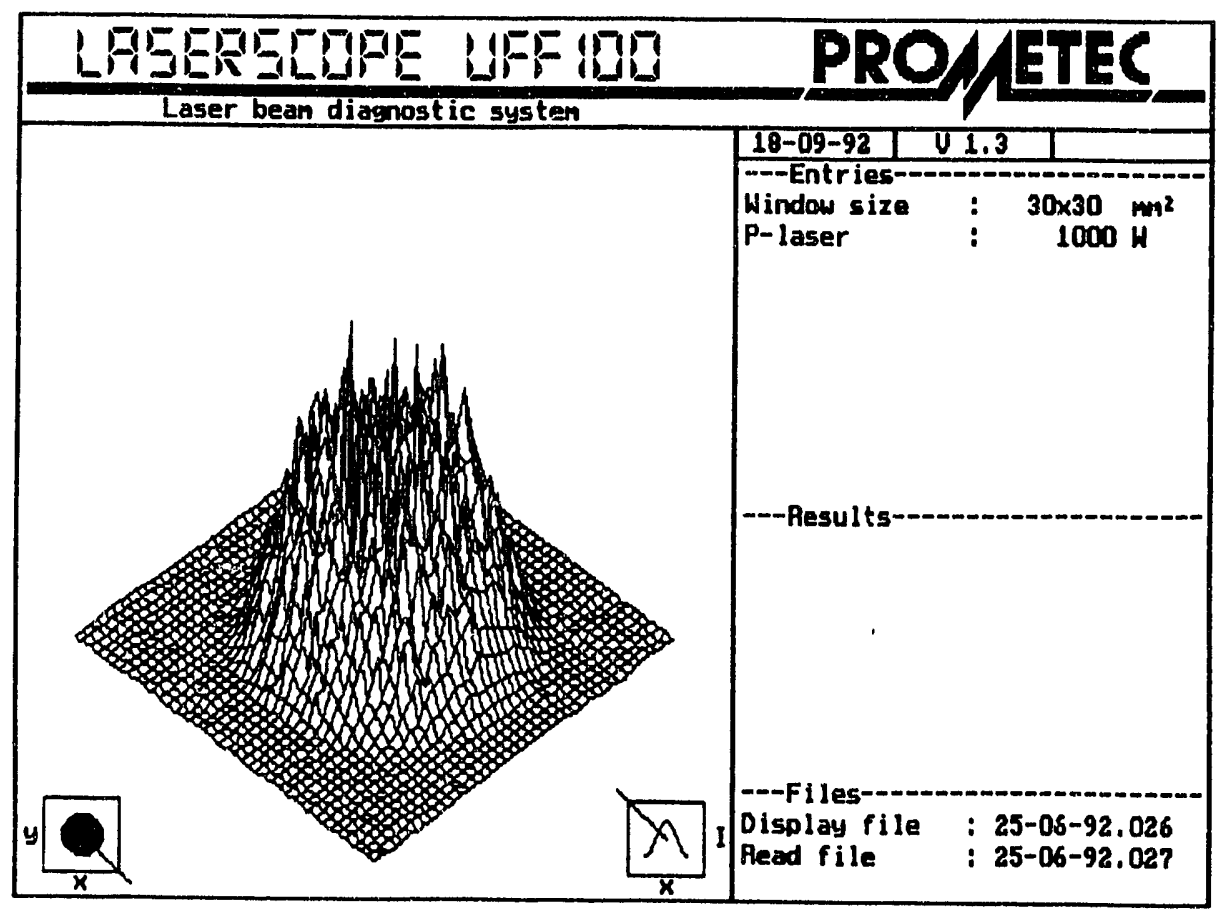

(b)

Fig. 5 Profile of collimated beam. (a) Beam area which contains $86 \%$ of beam energy and two orthogonal cross sections; (b) Isometric representation of beam intensity distribution. 


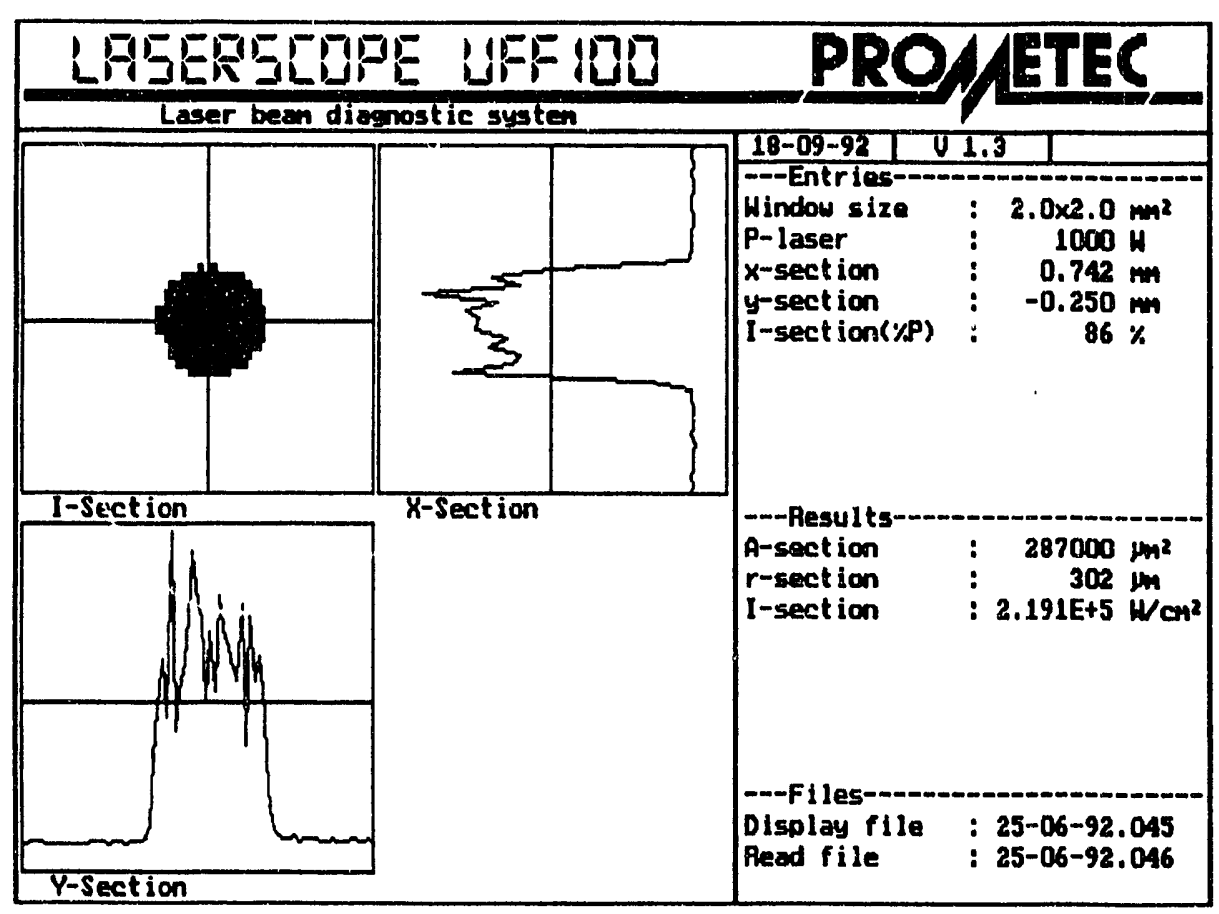

(a)

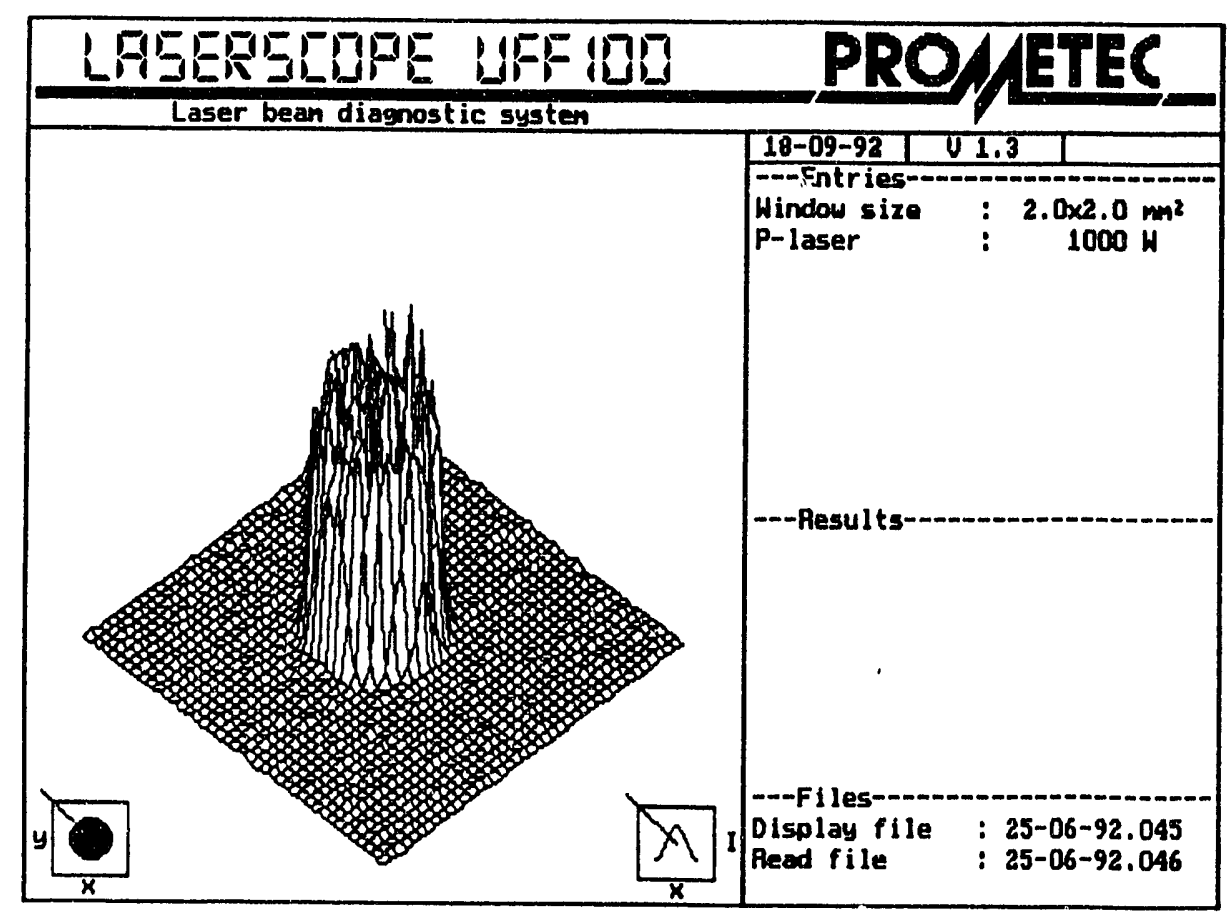

(b)

Fig. 6 Profile of focused beam. (a) Beam area which contains $86 \%$ of beam energy and two orthogonal cross sections; (b) Isometric representation of beam intensity distribution. 


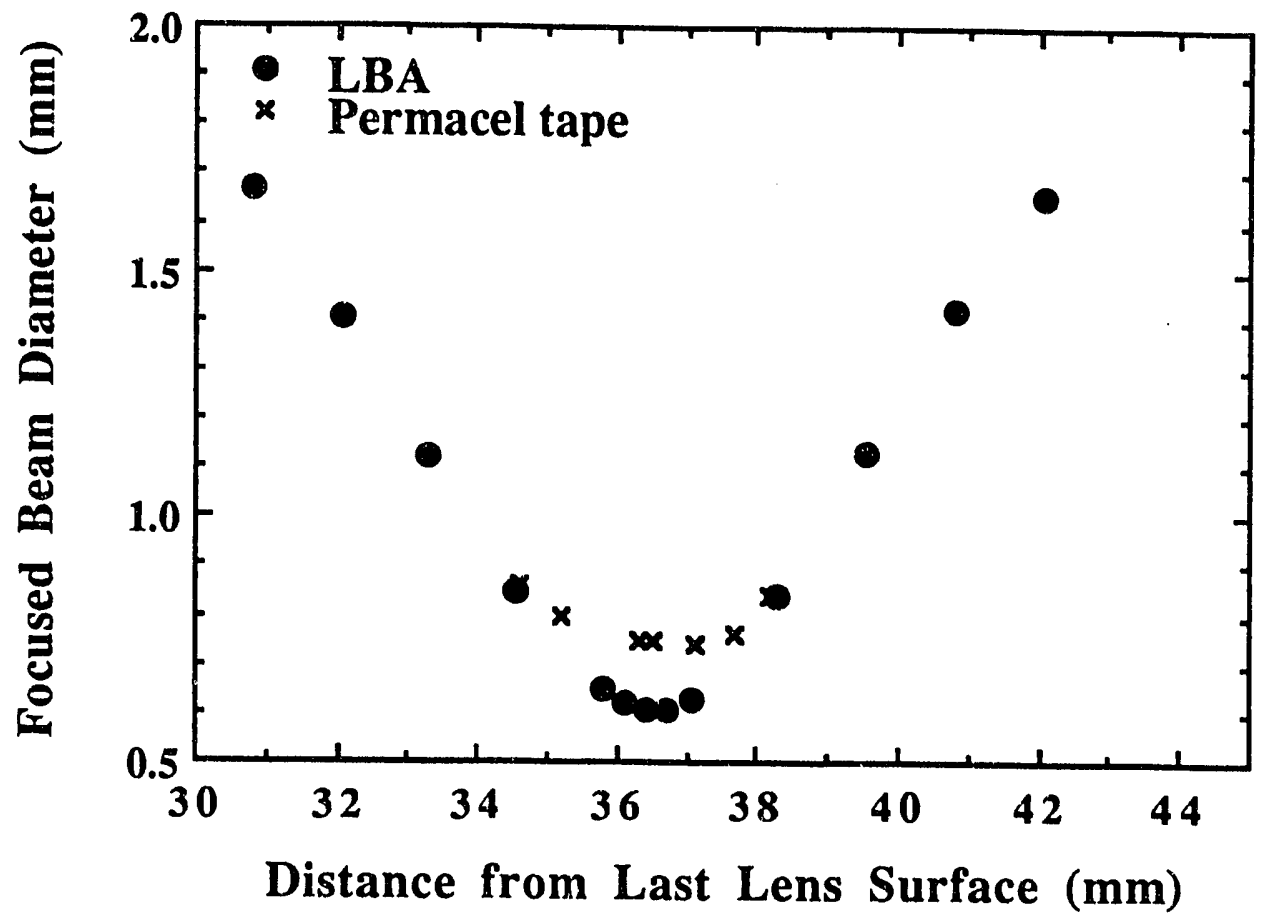

Fig. 7 Focused beam size in the vicinity of focal plane

\section{Conclusion}

The data presented demonstrate the capability of the Prometec LBA in characterizing the unfocused and focused beams of a pulsed kilowatt power YAG laser. Appropriate pulse width and pulse frequency are required for such measurements to allow synchronization with the profiling speed of the LBA. Profiles were measured for beams exiting the fiber, collimated beams from a beam collimator, and the focused beams. Various parameters such as beam diameter, beam divergence, focused beam size, focal length and power density can then be determined accurately. These parameters are important for the process development as well as the beam delivery optics design.

\section{References}

1. Hecht, J. (April, 1992). Laser Focus World, Vol.28, No. 4: 77-94.

2. Hoult, A. P. (May, 1989). Proc. 6th International Conf. on Lasers in Manufacturing, 23-30.

3. Sepold, G., Juptner, W. P. O. and Telepski, J. (1986). High Power Lasers and Their Industrial Applications, SPIE Vol. 650: 167-169.

4. Gilse, J. V., Koczera, S. and Greby, D. (January, 1991). Laser Beam Diagnostics, SPIE Vol. 1414: 45-54.

5. Fleischer, J. V.(January, 1991). Laser Beam Diagnostics, SPIE Vol. 1414:2-11.

6. Proposal for a Working Draft of ISO/TC 1/2/SC 9/WG 1, (May 29, 1992). 

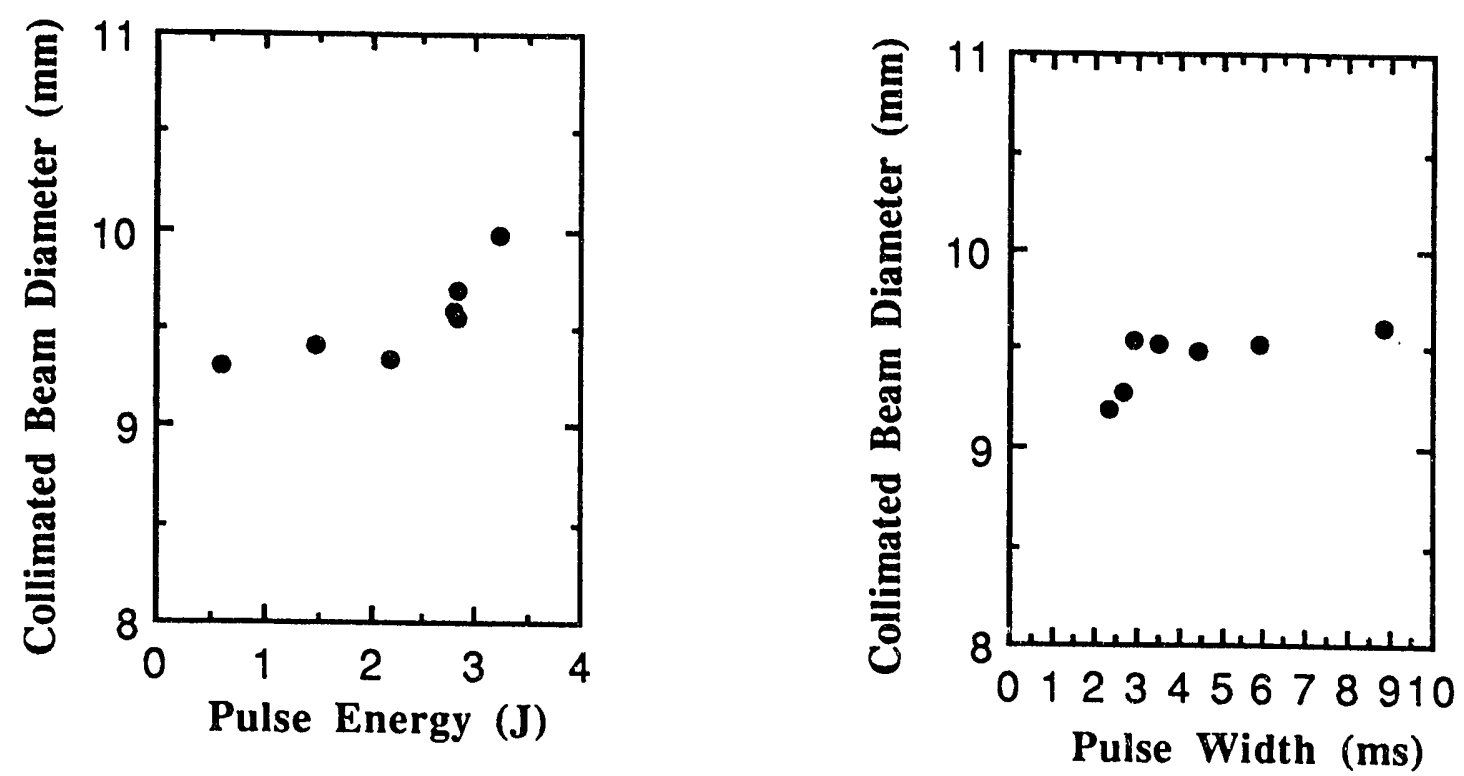

Fig. 8 Collimated beam diameter dependence on beam pulse energy and pulse width.

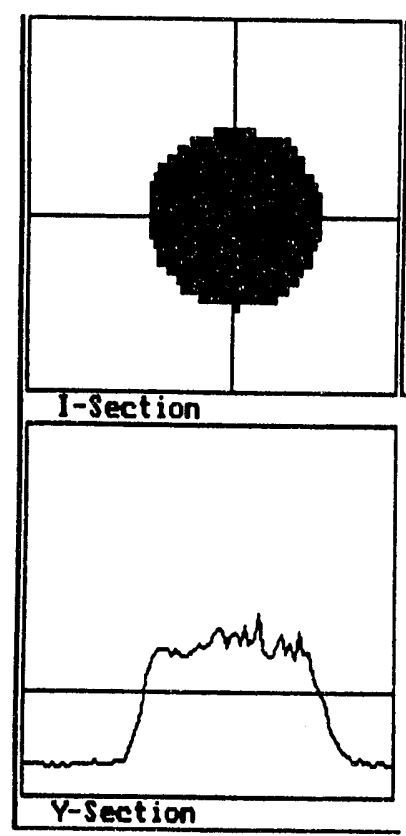

Top Hat Mode
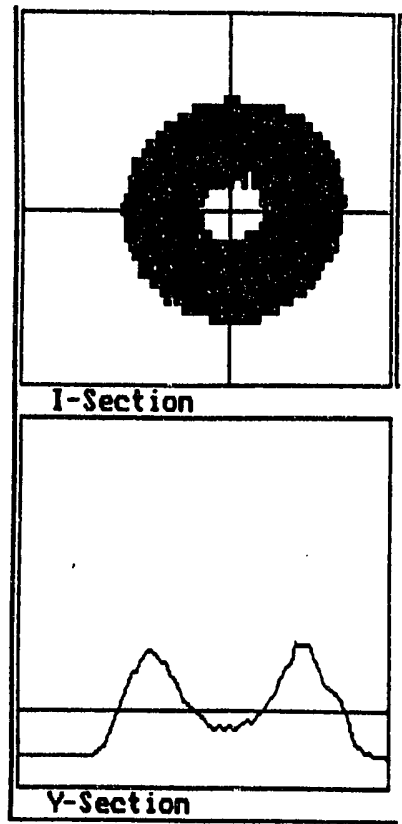

Doughnut Mode

Fig. 9 Two beam profiles from the old version of the YAG laser. 

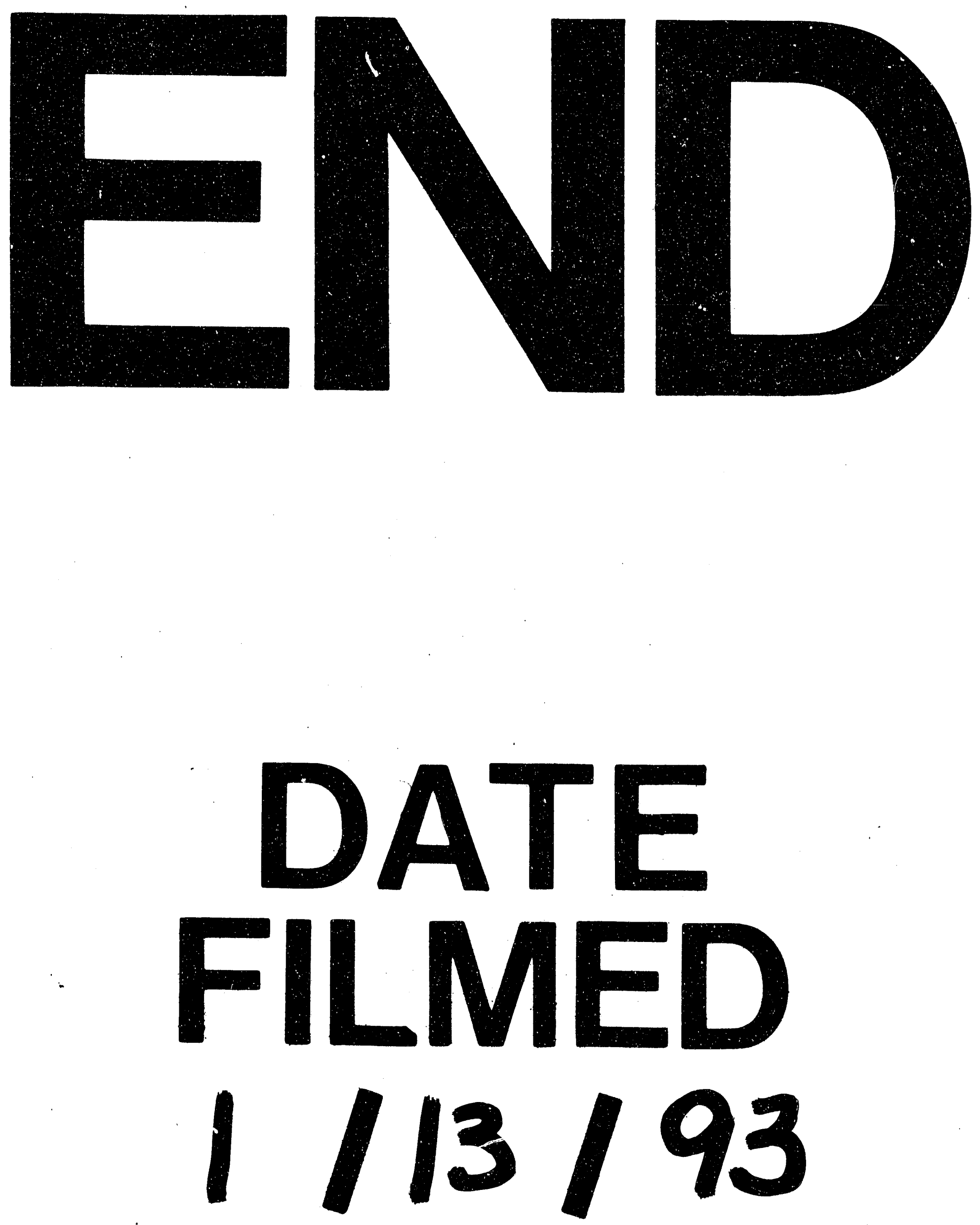